\title{
What Can Animal Memory Study Bring to the Assessment of Memory and Cognitive Skills for Intellectual Disability?
}

\author{
Fabienne Giuliani • Sophie Pernier • Jérôme Favrod • \\ François Grasset • Françoise Schenk
}

Published online: 15 January 2009

(C) Springer Science + Business Media, LLC 2009

\begin{abstract}
Three case studies are presented to investigate the possibility of evaluating memory and cognitive capacities of severe intellectual disability with attention given to the ecological environment. Two 22-year-old male patients and a 27-year-old male patient, all three with severe intellectual disability with no verbal communication skills, were evaluated with a new and original paradigm adapted to study cognition in humans from experimental paradigms. We developed a test based on animal models to complement the "home" scale of the Adolescent and Adult Psychoeducational Profile (AAPEP), an assessment instrument designed for adolescents and adults with severe developmental disabilities. Results show that the new instrument is helpful, not only to staff members who can better understand the poor performances of their patients in daily life activities but also in the elaboration of individual acquisition plans. These preliminary results demonstrate the interest in developing a larger controlled study and in publishing our procedure.
\end{abstract}

Keywords Intellectual disabilities · Cognitive behavior - Learning disability $\cdot$ Careers

The American Association on Mental Retardation describes intellectual disability as a particular state of functioning that begins in childhood and is characterized by limitation in both intelligence (Wechsler 1991, 1997) and adaptive skills. Intelligence refers to a

F. Giuliani · F. Grasset • F. Schenk

Institute of Psychology, University of Lausanne, 1015 Lausanne, Switzerland

F. Giuliani $(\bowtie) \cdot$ S. Pernier $\cdot$ F. Grasset

Consultation of Liaison Psychiatry for Mental Retardation Department,

Community Psychiatry Service, CHUV, Site de Cery, 1008 Prilly, Switzerland

e-mail: fabienne.Giuliani@unil.ch

J. Favrod

Rehabilitation Unit, Community Psychiatry Service, CHUV, Site de Cery, 1008 Prilly, Switzerland

F. Schenk

University and Psychiatric Neurosciences Center, Site de Cery, 1008 Prilly, Switzerland 
general mental capability. It involves the ability to reason, plan, solve problems, think abstractly, comprehend complex ideas, learn quickly and learn from experience.

In psychopathological diagnosis, a correct classification of a patient's level of intellectual disability is of fundamental importance to guarantee appropriate intervention for psychiatric problems suited to the patient's cognitive abilities. However, there is currently no cognitive evaluation adapted to severe intellectual disability.

Besides the limitations of intellectual disability diagnoses, reluctance of psychologists to diagnose low scoring as intellectual disability (Kanaya et al. 2003) and factors such as systematic fluctuations in IQ can be noted as: "inhibitors in the correct provision of intervention." According to predictions from the Flynn effect in intellectual disability in "Do we really have a criterion of mental retardation?": "The definition of mental retardation offered by the American Association on Mental Deficiency refers to an IQ of approximately 70 or below. This is identical to the Wechsler criterion of a test performance two standard deviations (SDs) below the population mean. In fact, Wechsler tests have not supplied such a criterion; rather they have deviated from it by anything from .27 to a full SD. Having done without such a criterion for 40 years, we should consider exchanging it for one that is fixed in time and whose external validity is attested to by an accumulated body of evidence " (Flynn 1985). For Kanaya et al. (2003) the impact of this IQ shift-in the absence of any real shift in the students' actual cognitive ability-resulted in large shifts in intellectual disability classifications. Explanation for this trend ranged from broader dissemination of research focusing on the negative impacts of labeling (Burke 1975; Edgerton 1967; Mercer 1973).

In addition, a number of studies (Bielecki and Swender 2004; Kober and Eggleton 2005; Kraemer et al. 2003; Salkever 2000; Stephens et al. 2005; Wehman et al. 1998; Wehmeyer 1994) demonstrates that the social competence of individuals with intellectual disability can be enhanced by social skills training. However, prior to designing effective training, an accurate assessment of adaptive capacities must be conducted. Cognitive assessment in intellectually disabled persons is important in planning appropriate mental health services. It is interesting (or important) to search for specific differences in cognitive strengths among different mentally retarded persons (note: "retarded" is an outdated word, now commonly used as an insult. Try "disabled" or "handicapped" or "impaired".

The limitations experienced by individuals with an intellectual disability often preclude traditional diagnostic evaluations. For example, individuals with an intellectual disability may: (a) not be able to adequately communicate their experiences, (b) display deficits in social skills that are mistaken for psychopathology, (c) show stress-induced maladaptive behaviors, and (d) exhibit increased severity of maladaptive behavior and cognitive delay (Sovner 1986). Finally, precise estimates in cognitive evaluation may also be limited by possible difficulties in verbal communication.

Evaluating the severely and profoundly mentally retarded population poses a particular challenge to the clinician in that the limitations described by Sovner (1986) are greater. When patients have little or no verbal communication skills, examiners may tend to attribute it to low global cognitive functioning without seeking the residual capacities (Vicari et al. 2001). From a theoretical point of view, it is of great relevance to find out what possible interaction takes place between impaired cognitive development and behavioral disorders of attention, 
mood, personality, and thought processes. From the practitioner's perspective, early and accurate differential diagnosis as well as intervention may have a profound impact on the success of the rehabilitative processes. Intellectual disability involves strengths and limitations in cognitive adaptive functioning that may coexist with or lead to impairment in emotional and social role functioning. In mental health, the inappropriate use of cognitive abilities is a primary component of psychiatric disorders (Di Nuovo and Buono 2007).

Sovner and Fogelman (1996), show that irritability is a persistent and unpleasant mood state characterized by low frustration tolerance, hostility, impulsivity, and aggression. It may be a normal response to stress or reflect significant central nervous system (CNS) dysfunction, i.e. dementia. Evidence from animal and human studies indicates that pathological irritability reflects limbic system dysfunction with a central dysregulation of serotoninergic inhibition of dopaminergic activity (Sovner and Fogelman 1996). In a mentally retarded person, irritability may lead to aggression and self-injury, particularly in an inappropriate environment. In addition, typical hyposomnia and pressured verbalizations associated with mania have been reported in patients with irritability. Clearly, there is a better chance of a correct diagnosis when the environment is appropriate!

It is of primary importance therefore to develop a cognitive task specific to severe intellectual disability. The most valuable innovations we want to introduce are: (1) to simplify the cognitive evaluation (2) to create a new and original paradigm developed from animal models to evaluate memory and cognitive capacities in severe intellectual disability and not to evaluate an impairment (3) to be ecological (4) to avoid the use of language abilities.

We assume that this new evaluation adapted for severe intellectual disability will encourage appropriate diagnoses. The careful evaluation of the residual capacities in severe intellectual disability will allow a positive socio-educational approach that will improve the adaptive behavioral skills, which in turn will reduce the stress factors induced by inappropriate behaviors.

Our approach consists of using the AAPEP (Mesibov et al. 1989) designed to measure the most crucial aspects of severe developmental handicaps (perception, fine and gross motor skills, eye-hand integration, cognitive performance and verbal performance). This program has been successful in accomplishing this by minimizing the use of language. We used in addition to the AAPEP a complement that is inspired from experiments in spatial orientation and memory and should help avoiding the traps of using IQ records. Several species (mammalian species including rats, nonhuman primates and humans) have similar visuospatial capacities (spatial learning and memory). The aim is to respond to specific adaptive behavior skills. No verbal communication is required and the subjects' knowledge about the environment is assessed from movement decisions and displacements, whether encouraged by curiosity or by reinforcement expectation.

Although not necessarily verbal, spatial memory is considered as a precursor of man's episodic memory and is easily tested in human subjects (adults or children) using controlled conditions similar to those used in animal experiments (Grobéty et al. 2000; Rossier et al. 2000; Schenk 1989). Spatial memory relies on high level cognitive capacities in animals and in humans and it requires the processing and integration of separately experienced events. Use of alternative automatic strategies is easy to detect 
since such dissociations have been well documented in neurophysiological research (Freedman and Oscar-Berman 1986a). However, such automatisms can also be encouraged to improve the subject's performance. Similar methods have also been used to study cognitive impairment in patients suffering from Down's syndrome (Nelson et al. 2005), Alzheimer's disease (Freedman and Oscar-Berman 1989), Parkinson's disease (Freedman and Oscar-Berman 1986b), and Korsakoff syndrome (Oscar-Berman and Bonner 1985; Oscar-Berman et al. 1992; Oscar-Berman and ZolaMorgan 1980). Nelson et al. (2005) tested adults with Down's syndrome in object discrimination learning, reversal learning and spatial memory tasks adapted from the protocols used to assess cognitive decline in canines and primates. These authors consider that the results obtained from these and possibly other tasks may serve as measures for clinical intervention trials in adults with intellectual disability.

Place learning tasks are designed and used to test the subject's (animal or human) capacity to learn "a place" while processing relations between distant landmarks. In this way, a "place" is a cognitive concept, demonstrating the capacity for a certain type of abstraction. Some authors postulate that place learning tasks bear on reference memory (Rasmussen et al. 1989). In this sense it is a division of long term memory. According to Olton (1983, p. 337): reference memory "contains general information about the rules and procedures that is applicable to many different instances of the same class of events, and thus, does not require the current instance of the class to be distinguished from any other instance of the same class." Foraging in a familiar or new environment (such as collecting items in a supermarket) allows working memory capacity to be assessed. Indeed, optimal efficacy requires the avoidance of recently visited places that must have lost interest, either because they were empty, or because the subject has already taken away their content. Spatial memory requires a convergence of the neural pathways originating from each eye onto common memory sites (Rudy et al. 1987) and finally spatial memory isn't only learning but also long-term memory retention (Spreng et al. 2002); contextual processing (Gluck and Myers 2001); episodic memory (Burgess et al. 2002); and relation processing (Eichenbaum et al. 1999). For example, Eichenbaum et al. proposed that spatial learning is an application case of its function for a more general learning process, the learning of relations. Here, spatial representation contributes to the general of "linking events within episodes". As a consequence, this memory space codes spatial and nonspatial relations among events, processing spatial relations for navigation and serial relations for solving more abstract nonspatial stimulus relations, such as those found in transitive inference (Dusek and Eichenbaum 1997).

\section{Method}

Setting

We selected three patients treated by the team of the outpatient liaison psychiatry consultation for the intellectually disabled of the Community psychiatry service of the Department of Psychiatry in Lausanne, Switzerland (DCPHM). Prior to participation in the evaluation, written consent was obtained from their legal representative(s) and the study was approved by the local research and ethics committee (protocol nb 48/08). 
Subjects

Three patients with severe intellectual disability coupled with non verbal have participated in the study:

Patient 1 is a 22-year-old male, living in his family's home.

Patient 2 is a 27-year-old male, living in a unit belonging to a specialized institution. Patient 3 is a 22-year-old male, living in a unit belonging to a specialized institution.

\section{AAPEP Procedure}

The Adolescent and Adult Psychoeducational Profile (AAPEP) (Mesibov et al. 1989) was administered to evaluate the level of developmental handicaps with only the scale called "Home." The AAPEP tests the six areas thought to be most important for successful, semi-independent functioning of severely handicapped individuals in the home. They include Vocational skills which are specific skills, such as sorting, matching, counting and using tools, which are tools that are necessary for the completion of typical vocational tasks. Independent functioning is a broad term covering the areas of self-help and self-guidance and includes skills like grooming, eating, toileting, using transportation independently, and following a schedule. Leisure skills allow one to use non-work time in a socially acceptable manner and include solitary or cooperative games, listening to music, and simple gross motor activities. Vocational behavior identifies and evaluates a patient's workrelated behaviors such as distractibility, the ability to work independently, the ability to sustain work and the ability to correct errors. Functional communication measures the degree to which one can understand instructions, respond to commands and prohibitions, communicate basic needs and use basic concepts. Interpersonal behavior refers to the abilities of working in groups, responding to the presence of others, and interacting appropriately. For each of these function areas, items are assessed through parent and nurse interviews. Each scale gives a score between zero and eight. (Subject/scoring of AAPEP). (Table 1)

Table 1 Summarizes the subjects' characteristics




Testing Apparatus and Instructions

The testing apparatus was adapted to study cognition in humans from experimental paradigms. The homing procedure (Schenk 1989), uses a circular arena which takes advantage of the tendency in rodents to return home. We have developed a simple paradigm of asking the tested subject to finding something, the stimulus, on a circular table, covered with plastic cups. This setting can be arranged in few minutes in a familiar room, so as to avoid any stress-induced reduction in capacity. Our strategy is to use a common basic reference task with a difficulty level that can be slowly increased depending on the subject's capacity. The method is ideal for the study of cognitive capacities in adults with severe intellectual disability because it doesn't require intact language abilities for administration and it relies on simple motivations. The advantage of these methods in the present project are that: (1) we have several years experience in establishing similar experiments for animals and humans, (2) the procedure is fast (30 min), unlike the standard test (between $2 \mathrm{~h}$ and $4 \mathrm{~h}$ ), (3) it can be made ecologically relevant depending on the "motivation being encouraged" and 4) it may be proposed as a game, thus more rewarding. We will also test mentally retarded patients in tasks bearing on cognitive mapping strategies, or working memory and temporal discontinuity disturbances. The test apparatus consisted of a portable linoleum structure, $80 \mathrm{~cm}$ in diameter. Over it, we put six blue goblets approximately $7 \times 10 \mathrm{~cm}$ in diameter (Fig. 1). Subjects were seated midline in front of the test apparatus and the experimenter sat opposite the subject. The stimulus used in the study was a piece of chocolate.

Standard verbal instructions were read to subjects prior to beginning each task. (i.e., "this is a game. In front of you, I am hiding a little chocolate under one of these goblets. Your job is to find the chocolate. Go ahead". If the subject appeared confused, the experimenter stated, "You can pick one". On successfully trial, verbal praise was also administered (e.g., "that's great!" and the reward (i.e., chocolate) was given to the subject. On trials with incorrect responses, the experimenter stated "No, the chocolate isn't (t)here, try again". Subjects were permitted unrestricted time to respond. The session duration was a maximum of $1 \mathrm{~h}$.
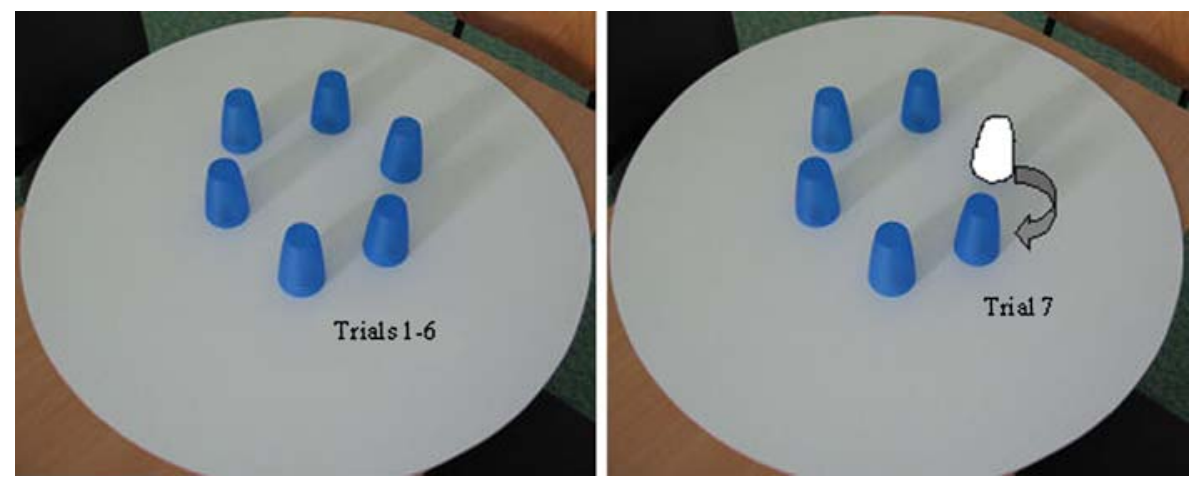

Fig. 1 Testing apparatus used to assess cognitive function in adults who have an intellectual disability 


\section{Spatial Discrimination}

The test subject is required to find the cup at the right place (to find the cup or "to find the chocolate hidden under one of the cups". This can be asked immediately after the experimenter has hidden the reinforcement, or following diverse interruptions aimed at revealing attention or memory impairments. At a higher level, capacities for short cut and detour from different start (adequate "ego or allocentred" decisions) may reveal cognitive resources not evident from verbal questions. (Table 2)

Two kinds of behavioral measures were taken. First, the number of goblets lifted until the patient finds the piece of chocolate was recorded as an indication of working memory. Second, the type of error was assessed from each trial according to the precedent trial as well as current difficulty. Step 1 (describes following up) indicates working memory and reference memory capacities. Step 2 evaluates the episodic memory and visuospatial capacities. (Table 3)

\section{Results}

Figure 2 shows results of the AAPEP for the three subjects. It can be noticed that patient 1 showed the highest scores (average=3.66), especially regarding « leisure skills » and « interpersonal behavior ». Patients 2 (average $=2.33$ ) and 3 (average $=2.50$ ) show little competence from the point of view of the Psychoeducational Profile.

Figure 3 shows the results from the spatial test for the three subjects. Subject 1 has succeeded in all trials without errors. Patients 2 and 3 have made several mistakes that will now be described.

Subjects 2 and 3 succeeded in trials 1 and 2 without errors. This confirms that the patients understood the game. In trial 3, when there was a little interference before the answer (drink a glass of water) subject 2 made a mistake, revealing a certain failure in his working memory. The same subjects made several mistakes at trials $4\left(180^{\circ}\right.$ rotation $)$ and 5 ( $90^{\circ}$ rotation), being unable to find the spatial position of the reward. At trial 6 , when there was an interference activity of half an hour, subject 3 found the goblet containing the reward without failure. However, subject 2 failed as in trial 3 when he was invited to drink a glass of water before answering, which confirms his working memory deficit.

In trial 7, when the white goblet containing the reward had changed position before the answer (without the subject knowing), all the subjects chose the white goblet independently of this change. After that, when subjects are asked for an alternative location where an additional reward could be eventually placed, the three of them lifted the blue goblet situated in the former position of the white one.

Table 2 Description of the evaluation

Working memory 
Table 3 Describes the flow chart of all trials



Table 4 describes the result obtained with each patient. To obtain a result in percentage terms, we have transformed the level of developmental handicaps $(0=$ high developmental handicaps; $8=$ low developmental handicaps $)$ in successful $(0=0 \% ; 1=$ $12.5 \% ; 8=100 \%$ of successful). For the spatial discrimination, we proceeded in the same manner. $100 \%$ success represented no error, with one error, the patient obtained $83.3 \%$ success, with two errors, the patient obtained $66.6 \%$ success, three errors, $50 \%$ success, four errors, $33.3 \%$ success, five errors, $16.6 \%$ success. 

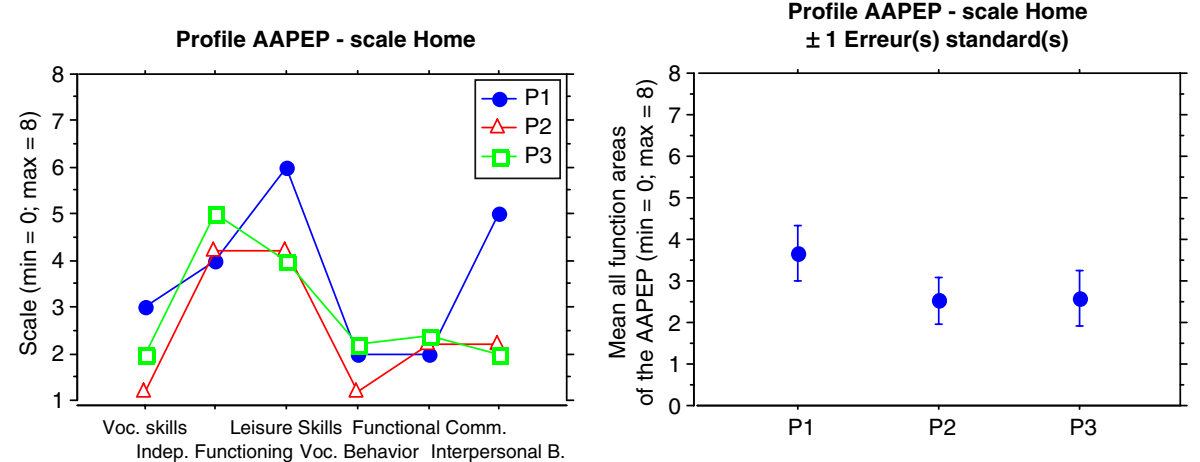

Fig. 2 The Adult Psychoeducational Profile (AAPEP). On the left, scale on six areas of the AAPEP; on the right, global measure on AAPEP

Fig. 3 Place discrimination during our cognitive task

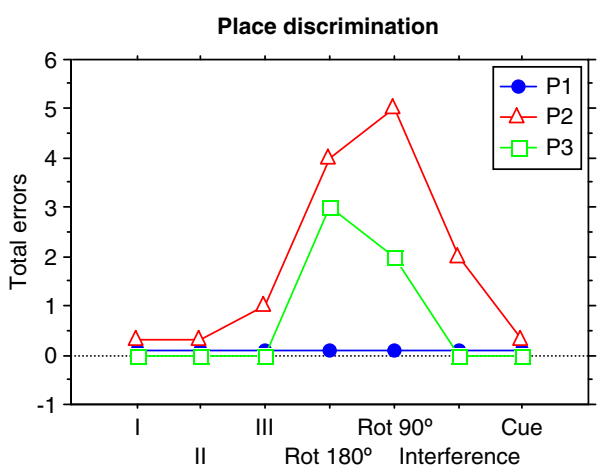

Table 4 High success represents a low developmental handicap

\begin{tabular}{llccr}
\hline & & Patient 1 & Patient 2 & Patient 3 \\
\hline \multirow{2}{*}{ AAPEP } & Vocational skills & 37.5 & 12.5 & 12.5 \\
& Independent functioning & 50.0 & 50.0 & 62.5 \\
& Leisure skills & 75.0 & 50.0 & 50.0 \\
& Vocational behaviour & 25.0 & 12.5 & 12.5 \\
& Functional communication & 25.0 & 25.0 & 25.0 \\
& Interpersonal behaviour & 62.5 & 25.0 & 25.0 \\
Spatial discriminate. & Working memory & 100.0 & 94.4 & 100.0 \\
& Episodic memory & 100.0 & 66.6 & 100.0 \\
& Reference memory & 100.0 & 100.0 & 100.0 \\
& Contextual processing & 100.0 & 25.0 & 58.3 \\
& Long term memory & 100.0 & 66.6 & 100.0 \\
& Average of successful & 70.45 & 47.96 & 58.71 \\
\hline
\end{tabular}




\section{Discussion}

The three cases described here, suggest that an evaluation test which avoids use of language could be integrated into the clinical routine. As it was shown by Mesibov et al. (1989), the AAPEP doesn't reveal a significant difference when compared to IQ tests or to results obtained from adults presenting developmental disability and those diagnosed as autistic. However, the AAPEP is a tool that allows to specifically evaluate patients with severe to medium intellectual disability. Our results were slightly inferior to those reported by Mesibov et al. (1989) for over 60 subjects (autistic and non-autistic). Indeed, the study reported an average of 3.86 while our study presents an average of 3.01 for three subjects. Mesinov et al. state that the problem of AAPEP is that it takes a lot of time. In their paper they mentioned an approximate duration of $31 / 2 \mathrm{~h}$, which is somewhat long. The advantage of our evaluation is that we use only the home scale AAPEP with our innovative evaluation. The test takes only two periods of $30 \mathrm{~min}$. Performances found with the AAPEP and with our cognitive evaluation were compared. It can be noticed that patient 1 obtained the better scores with the AAPEP and that he also succeeded completely in the cognitive evaluation. That means a good working and episodic memory as well as a satisfying decentration capacity (or a capacity to relate proprioceptive elements with the environment). In light of the AAPEP, patient 3 shows little competence and professional behavior (score of 1) and, regarding cognitive evaluation, he shows a deficit in working memory. This deficit can explain his difficulties with the AAPEP with consequences for his working capacities. Successive trials dealing with the subjects' spatial position changes before finding the reward showed that subjects 2 and 3 are not capable of successively adjusting mental images. Finally, regarding the indexed trial, the experiment revealed the significant importance of the color index. In fact, the three subjects managed to follow the index in spite of its change in spatial position.

In summary, the AAPEP and the memory procedure can facilitate the development of appropriate individualized programs based on the evaluation while reducing irritability and hyperactivity by more empathetic behavior toward the patients and being sensitive about using appropriate environments. The important finding is that this test was useful to help the clinical team to adjust its intervention with the psychopathological problem. Moreover, for patient 1 , the evaluation in particular made it possible to find a specialized institution for him and we also could share with his parents, that in spite of an absence of language, he had great fine and gross motor skills, eye-hand integration, attention, working and episodic memories. Therefore, a more productive activity had to be found in order to control irritability and hyperactivity. This activity should not be too repetitive and should take into account his fine and gross motor skills and spatial memory (e.g., swimming pool, running, to help in the kitchen, to set the table, to clean). For patients 2 and 3, the evaluation allowed a positive feedback of professional careers because we could put forward attention deficits but also suggest the use of systematic spatial and temporal cues that allowed compensating for the patients cognitive deficits.

The preliminary results presented here provide important information about how to design a larger controlled study of cognitive skills in intellectual disability using a non-verbal task. Of course, this protocol is under 
development in order to add other cognitive functions to it (e.g., executive functioning). Our future research is aimed not only at the improvement of diagnosis and individualized handicap profiles, but also at supporting teaching methods and cognitive and behavior therapy. These preliminary results demonstrate the interest in developing a larger controlled study.

\section{References}

Bielecki, J., \& Swender, S. L. (2004). The assessment of social functioning in individuals with mental retardation: a review. Behavior Modification, 28(5), 694-708. doi:10.1177/0145445503259828.

Burgess, N., Maguire, E. A., \& O'Keefe, J. (2002). The human hippocampus and spatial and episodic memory. Neuron, 35(4), 625-641. doi:10.1016/S0896-6273(02)00830-9.

Burke, A. A. (1975). Placement of black and white children in educable mentally handicapped classes and learning disability classes. Exceptional Children, 41(6), 438-439.

Di Nuovo, S. F., \& Buono, S. (2007). Psychiatric syndromes comorbid with mental retardation: differences in cognitive and adaptive skills. Journal of Psychiatric Research, 41(9), 795-800. doi:10.1016/j.jpsychires.2006.02.011.

Dusek, J. A., \& Eichenbaum, H. (1997). The hippocampus and memory for orderly stimulus relations. Proceedings of the National Academy of Sciences of the United States of America, 94(13), 71097114. doi:10.1073/pnas.94.13.7109.

Edgerton, R. B. (1967). The cloak of competence: Stigma in the lives of the mentally retarded. Berkeley, California: University of California press.

Eichenbaum, H., Dudchenko, P., Wood, E., Shapiro, M., \& Tanila, H. (1999). The hippocampus, memory, and place cells: is it spatial memory or a memory space? Neuron, 23(2), 209-226. doi:10.1016/S08966273(00)80773-4.

Flynn, J. R. (1985). Wechsler intelligence tests: do we really have a criterion of mental retardation? American Journal of Mental Deficiency, 90(3), 236-244.

Freedman, M., \& Oscar-Berman, M. (1986a). Bilateral frontal lobe disease and selective delayed response deficits in humans. Behavioral Neuroscience, 100(3), 337-342. doi:10.1037/0735-7044.100.3.337.

Freedman, M., \& Oscar-Berman, M. (1986b). Selective delayed response deficits in Parkinson's and Alzheimer's disease. Archives of Neurology, 43(9), 886-890.

Freedman, M., \& Oscar-Berman, M. (1989). Spatial and visual learning deficits in Alzheimer's and Parkinson's disease. Brain and Cognition, 11(1), 114-126. doi:10.1016/0278-2626(89)90009-2.

Gluck, M. A., \& Myers, C. E. (2001). Gateway to memory: An introduction to neural network modeling of the hippocampus and learning. Cambridge Mass: Cambridge.

Grobéty, M. C., Morand, M., \& Schenk, F. (2000). Cognitive mapping in rats and humans : the tent-maze, a place learning task in visually disconnected environments. In S. O'Nuallain (Ed.), Spatial Cognition. Foundations and applications: Spatial cognition. Foundations and applications (vol. Vol. 26, (pp. 105-126)). Amsterdam: John Benjamins.

Kanaya, T., Ceci, S. J., \& Scullin, M. H. (2003). The rise and fall of IQ in special ed: Historical trends and their implications. Journal of school psychology, 41, 453-465.

Kober, R., \& Eggleton, I. R. (2005). The effect of different types of employment on quality of life. Journal of Intellectual Disability Research, 49(Pt 10), 756-760. doi:10.1111/j.1365-2788.2005.00746.x.

Kraemer, B. R., McIntyre, L. L., \& Blacher, J. (2003). Quality of life for young adults with mental retardation during transition. Mental Retardation, 41(4), 250-262. doi:10.1352/0047-6765(2003) 41<250:QOLFYA $>2.0 . C O ; 2$.

Mercer, J. R. (1973). Labeling the mentally retarded. Berkeley, California: University of California press.

Mesibov, G. B., Schopler, E., \& Caison, W. (1989). The Adolescent and Adult Psychoeducational Profile: assessment of adolescents and adults with severe developmental handicaps. Journal of Autism and Developmental Disorders, 19(1), 33-40. doi:10.1007/BF02212716.

Nelson, L., Johnson, J. K., Freedman, M., Lott, I., Groot, J., Chang, M., et al. (2005). Learning and memory as a function of age in Down syndrome: a study using animal-based tasks. Progress in NeuroPsychopharmacology \& Biological Psychiatry, 29(3), 443-453. doi:10.1016/j.pnpbp.2004.12.009.

Olton, D. S. (1983). Memory functions and the hippocampus. In W. Seifert (Ed.), Neurobiology of the hippocampus (pp. 335-373). London: Academic. 
Oscar-Berman, M., \& Zola-Morgan, S. M. (1980). Comparative neuropsychology and Korsakoff's syndrome. II.-Two-choice visual discrimination learning. Neuropsychologia, 18(4-5), 513-525. doi:10.1016/0028-3932(80)90153-0.

Oscar-Berman, M., \& Bonner, R. T. (1985). Matching- and delayed matching-to-sample performance as measures of visual processing, selective attention, and memory in aging and alcoholic individuals. Neuropsychologia, 23(5), 639-651. doi:10.1016/0028-3932(85)90065-X.

Oscar-Berman, M., Hutner, N., \& Bonner, R. T. (1992). Visual and auditory spatial and nonspatial delayed-response performance by Korsakoff and non-Korsakoff alcoholic and aging individuals. Behavioral Neuroscience, 106(4), 613-622. doi:10.1037/0735-7044.106.4.613.

Rasmussen, M., Barnes, C. A., \& McNaughton, B. L. (1989). A systematic test of cognitive mapping, working memory temporal discontinuity theories of hippocampal function. Psychobiology, 17, 345-348.

Rossier, J., Grobéty, M. C., Spreng, M., \& Schenk, F. (2000). Impact of space configuration on route choices in humans and rats. In E. a. K. H. Thommen (Ed.), Comparer ou prédire : exemples de recherches en psychologie comparative aujourd'hui (pp. 37-52). Fribourg: Les Éditions Universitaires de Fribourg.

Rudy, J. W., Stadler-Morris, S., \& Albert, P. (1987). Ontogeny of spatial navigation behaviors in the rat: dissociation of "proximal"- and "distal"-cue-based behaviors. Behavioral Neuroscience, 101(1), 6273. doi:10.1037/0735-7044.101.1.62.

Salkever, D. S. (2000). Activity status, life satisfaction and perceived productivity for young adults with developmental disabilities. Journal of Rehabilitation, 66, 4-13.

Schenk, F. (1989). A homing procedure for studying spatial memory in immature and adult rodents. Journal of Neuroscience Methods, 26(3), 249-258. doi:10.1016/0165-0270(89)90123-4.

Sovner, R. (1986). Limiting factors in the use of DSM-III criteria with mentally ill/mentally retarded persons. Psychopharmacology Bulletin, 22(4), 1055-1059.

Sovner, R., \& Fogelman, S. (1996). Irritability and Mental Retardation. Seminars in Clinical Neuropsychiatry, 1(2), 105-114.

Spreng, M., Rossier, J., \& Schenk, F. (2002). Spaced training facilitates long-term retention of place navigation in adult but not in adolescent rats. Behavioural Brain Research, 128(1), 103-108. doi:10.1016/S0166-4328(01)00266-2.

Stephens, D. L., Collins, M. D., \& Dodder, R. A. (2005). A longitudinal study of employment and skill acquisition among individuals with developmental disabilities. Research in Developmental Disabilities, 26(5), 469-486. doi:10.1016/j.ridd.2003.12.003.

Vicari, S., Bellucci, S., \& Carlesimo, G. A. (2001). Procedural learning deficit in children with Williams syndrome. Neuropsychologia, 39(7), 665-677. doi:10.1016/S0028-3932(01)00012-4.

Wechsler, D. (1991). Wechsler intelligence scale for children-revised manual. New York: The Psychological Corporation.

Wechsler, D. (1997). Wechsler Adult Intelligence Scale (3rd ed.). San Antonio: The Psychological Corporation.

Wehman, P., Revell, W. G., \& Kregel, J. (1998). Supported employment: a decade of rapid growth and impact. American Rehabilitation, 24, 31-43.

Wehmeyer, M. L. (1994). Employment status and perceptions of control of adults with cognitive and developmental disabilities. Research in Developmental Disabilities, 15(2), 119-131. doi:10.1016/ 0891-4222(94)90017-5. 